\title{
LOCAL COMPLETENESS FOR EIGENFUNCTIONS OF REGULAR MAXIMAL ORDINARY DIFFERENTIAL OPERATORS
}

\author{
ROBERT CARLSON
}

\begin{abstract}
Let $L=\sum_{m=0}^{n} A_{m}(x) D^{m}$ be a differential operator on $\oplus^{k} L^{2}\{0,1]$ with infinitely differentiable $k \times k$ matrix valued coefficients. Assume that $\operatorname{det} A_{n}(x) \neq 0$ for $x \in[0,1]$. The domain of $L$ is the set of $k$-vector valued functions $f$ such that $f \in C^{n-1}\left([0,1), f^{(n-1)}\right.$ is absolutely continuous on $[0,1]$ and $L f \in \oplus^{k} L^{2}[0,1]$. Let $x_{0} \in(0,1)$. Then there is a neighborhood $U\left(x_{0}\right)$ containing $x_{0}$ such that the restrictions of the eigenfunctions of $L$ to $U\left(x_{0}\right)$ have dense span in $\oplus^{k} L^{2}\left[U\left(x_{0}\right)\right]$. The example $L=e^{-i x} d / d x$ shows that this is the best possible abstract result.
\end{abstract}

There is a very substantial literature concerning the completeness of eigenfunctions for ordinary differential operators; usually the operators considered are perturbations of some selfadjoint operator. When one allows the leading coefficient of the differential operator to be an arbitrary (smooth) matrix valued function it is possible to find examples of regular maximal operators whose eigenfunctions are not complete. It is then natural to ask whether the lack of a complete set of eigenfunctions for such operators is a local or global problem. Theorem 1 shows that the problem is global.

We consider ordinary differential operators $L=\sum_{m=0}^{n} A_{m}(x) D^{m}$ where $A_{m}(x)$ is an infinitely differentiable $k \times k$ matrix valued function on $[0,1]$. We assume that $\operatorname{det} A_{n}(x) \neq 0$ for $x \in[0,1]$, that $L$ acts on the Hilbert space $\bigoplus^{k} L^{2}[0,1]$, and that the domain of $L$ is the set of $k$-vector valued functions $f$ which satisfy $f \in$ $C^{n-1}([0,1]), f^{(n-1)}$ is absolutely continuous on $[0,1]$, and $L f \in \bigoplus^{k} L^{2}[0,1]$. Denote by $D(L), R(L), N(L)$ respectively the domain, range and kernel of the operator $L$. It is known that the domain of $L^{*}$, the adjoint of $L$, is the set of functions in $D(L)$ which also satisfy $f^{(m)}(0)=f^{(m)}(1)=0$ for $m=0, \ldots, n-1$. Many of the basic facts about these operators can be found in Goldberg [4], or in Coddington and Dijksma [2].

We call such an operator $L$ a regular maximal operator. Recall that for every complex number $\lambda$ the initial value problem

$$
L \Gamma(x, \lambda)=\lambda \Gamma(x, \lambda), \quad \tilde{\Gamma}(0, \lambda)=I_{n k}
$$

has a unique $k \times n k$ matrix valued solution. Here $I_{n k}$ is the $n k \times n k$ identity matrix and

Received by the editors March 9, 1979 and, in revised form, April 27, 1979 and July 9, 1979.

AMS (MOS) subject classifications (1970). Primary 47E05. 


$$
\tilde{\Gamma}(0, \lambda)=\left|\begin{array}{c}
\Gamma(0, \lambda) \\
\Gamma^{(1)}(0, \lambda) \\
\vdots \\
\Gamma^{(n-1)}(0, \lambda)
\end{array}\right| .
$$

We denote by $\gamma_{i}(\lambda)$ the $i$ th column of $\Gamma(x, \lambda) ; \gamma_{i}(\lambda)$ is an entire $\bigoplus^{k} L^{2}[0,1]$ valued function, and $\gamma_{i}(x, \lambda)$ is, for each $x \in[0,1]$, an entire $C^{k}$ valued function. The linear span of $\left\{\gamma_{i}(\lambda) \mid i=1, \ldots, n k ; \lambda\right.$ complex $\}$ will be denoted by $\mathcal{S}$. By $\mathcal{S}^{c}$ we mean the Hilbert space closure of $\mathcal{S}$, and by $\mathcal{S}^{\perp}$ the orthogonal complement.

Finally, we will use $\|\cdot\|$ to indicate the usual Hilbert space norm in $\bigoplus^{k} L^{2}[0,1]$ or $C^{n}$ induced by the inner product $(\cdot, \cdot)$, or the usual operator norm, depending on the context. We denote by $C$ the complex numbers.

THEOREM 1. Let $L=\sum_{i=0}^{n} A_{i}(x) D^{i}$ be a regular maximal operator. Suppose that $x_{0} \in(0,1)$. Then there is a neighborhood $U\left(x_{0}\right)$ of $x_{0}$ such that the eigenfunctions of $L$ restricted to $U\left(x_{0}\right)$ have dense span in $\bigoplus^{k} L^{2}\left[U\left(x_{0}\right)\right]$.

Before proving this we discuss some lemmas and related results. Let $\delta g\left(\lambda_{0}\right)$ be the linear span of $\left\{(\partial / \partial \lambda)^{l} \gamma_{i}\left(\lambda_{0}\right) \mid i=1, \ldots, n k ; l=0,1,2, \ldots\right\}$. The existenceuniqueness theory for linear ordinary differential operators shows that the equation $\left(L-\lambda_{0}\right)^{m} x=0$ has an $m n k$ dimensional set of solutions in $D\left(L^{m}\right)$, and they are exhibited in $\delta g\left(\lambda_{0}\right)$.

LeMMA 2. For all $\lambda_{0} \in C, S^{c}=\left[\delta g\left(\lambda_{0}\right)\right]^{c}$.

Proof. Let $g \in \bigoplus^{k} L^{2}[0,1]$ be orthogonal to $\gamma_{i}(\lambda)$ for all $\lambda \in C$ and $i=$ $1, \ldots, n k$. Then $\left(\gamma_{i}(\lambda), g\right)=0$, which implies $\left(\partial^{l} / \partial \lambda^{l}\right)\left(\gamma_{i}(\lambda), g\right)=\left(\left(\partial^{l} / \partial \lambda^{l}\right) \gamma_{i}(\lambda), g\right)$ $=0$. Since $\gamma_{i}(\lambda)$ is analytic we have

$$
\gamma_{i}(\lambda)=\sum_{l=0}^{\infty} \frac{\partial^{l} \gamma_{i}}{\partial \lambda^{l}}\left(\lambda_{0}\right) \frac{\left(\lambda-\lambda_{0}\right)^{l}}{l !}
$$

Thus if $\left(\left(\partial^{l} / \partial \lambda^{l}\right) \gamma_{i}\left(\lambda_{0}\right), g\right)=0$ for all $l,\left(\gamma_{i}(\lambda), g\right)=0$.

Corollary 3. The eigenfunctions of $e^{-i x} D$ do not have dense span in $L^{2}[0,2 \pi]$.

Proof. The eigenfunctions are $\gamma(\lambda)=\exp \left(\lambda \int_{0}^{x} e^{i s} d s\right)$. Now $\partial^{l} \gamma(0) / \partial \lambda^{l}=$ $\left[\int_{0}^{x} e^{i s} d s\right]^{l}=(-i)^{l}\left[e^{i x}-1\right]^{l}$. Hence the closed span of the eigenfunctions is the closed span of $e^{i l x}$ in $L^{2}[0,2 \pi], l=0,1,2, \ldots$ The orthogonal complement is the closed span of $e^{i l x}, l=-1,-2, \ldots$.

THEOREM 4. The codimension of $\mathcal{S}$ is either zero or infinite.

Proof. Since $\varsigma^{\perp}$ is orthogonal to $N(L), \varsigma^{\perp} \subset R\left(L^{*}\right)$. Let $f \in \varsigma^{\perp}$ and let $L^{*} g=f$. Suppose that $L \gamma=\lambda \gamma, \lambda \neq 0$. Then $(\gamma, g)=(\gamma \lambda, g) / \lambda=(L \gamma, g) / \lambda=$ $(\gamma, f) / \lambda=0$. By analyticity $(\gamma, g)=0$ for all $\lambda \in C$. Thus $\mathcal{S}^{\perp}$ is invariant under $\left(L^{*}\right)^{-1}$. If $\mathcal{S}^{\perp}$ were finite dimensional $\left(L^{*}\right)^{-1}$ would have an eigenvalue. However this is impossible by the basic uniqueness result for ordinary differential equations and the known characterization of $D\left(L^{*}\right)$. 
THEOREM 5. If $T$ and $L$ are regular maximal operators and $T L=L T$ then $\mathcal{S}_{T}^{c}=\mathcal{S}_{L}^{c}$ where $\mathcal{S}_{T}$ and $\mathcal{S}_{L}$ are the spans of the eigenfunctions of $T$ and $L$ respectively.

Proof. The eigenspaces of $L$ are invariant under $T$. Since they are finite dimensional they are spanned by generalized eigenfunctions for $T$. Using Lemma 2 we see that

$$
\begin{aligned}
& \delta_{L} \subset \operatorname{span}\left\{\cup_{\lambda \in C}(\delta g)_{T}(\lambda)\right\} \subset\left[\delta_{T}\right]^{c} \text { and } \\
& \delta_{T} \subset \operatorname{span}\left\{U_{\lambda \in C}(\delta g)_{L}(\lambda)\right\} \subset\left[\delta_{L}\right]^{c},
\end{aligned}
$$
so $\mathfrak{S}_{L}^{c}=\mathfrak{S}_{T}^{c}$.

Proof of Theorem 1. Let $V_{v}, \nu>0$, denote a matrix such that $V_{v}^{-1} A_{n}\left(x_{0}\right) V_{p}$ is in Jordan normal form with the elements of the first superdiagonal being either 0 or $\nu$. Note first that $L$ and $\left[V_{\nu}^{-1} L V_{\nu}\right]^{j}$ have dense span simultaneously for any positive integer $j$. Write the diagonal elements of $V_{v}^{-1} A_{n}\left(x_{0}\right) V_{\nu}$ as $r_{l} e^{2 \pi i \theta_{l}}, l=1, \ldots, k$, where $\theta_{l}$ and $r_{l}$ are real. Theorem 201 of Hardy and Wright [5] asserts that there is an integer $j$ such that for any $\varepsilon>0$ the numbers $j \theta_{1}, \ldots, j \theta_{k}$ differ from integers by less than $\varepsilon$, so the numbers $r_{l}^{j} e^{2 \pi i j \theta_{1}}$ can be made to lie in a sector of the form $\{z|| \arg z \mid<\varepsilon\}$ for any $\varepsilon>0$. For our purposes $\varepsilon=\pi / 10$ will do. Since the diagonal matrix $F$ with entries $r_{l}^{j} e^{2 \pi i j \theta_{l}}$ satisfies $\operatorname{Re} F=\frac{1}{2}\left(F+F^{*}\right)>c I, c>0$, the same is true by continuity for the matrices $\left[V_{\nu}^{-1} A_{n}\left(x_{0}\right) V_{\nu}\right]^{j}$ for $\nu$ sufficiently small. Thus, without loss of generality we can assume that $L$ has order $2 r, r$ even, and $A_{2 r}\left(x_{0}\right)$ satisfies $\operatorname{Re} A_{2 r}\left(x_{0}\right)>c I, c>0$.

Write

$$
L=D^{r} A_{2 r}(x) D^{r}+\sum_{m=0}^{r-1}\left\{D^{m+1} B_{m}(x) D^{m}+D^{m} G_{m}(x) D^{m}\right\} .
$$

Since $\operatorname{Re}\left(A_{2 r}\left(x_{0}\right)\right)>c I>0$ for some $c>0$, the continuity of $A_{2 r}$ implies that the same inequality is valid for $x$ in some open interval $U\left(x_{0}\right)$ containing $x_{0}$. Let $f$ be an $n=2 r$ times continuously differentiable $k$-vector valued function with support in $U\left(x_{0}\right)$. We claim that there are positive constants $\mu, c_{1}, c_{2}$ such that, for all $f$, $\operatorname{Re}([L+\mu I] f, f)>c_{1}\|f\|^{2}$ and $|\operatorname{Im}([L+\mu I] f, f)|<c_{2} \operatorname{Re}([L+\mu I] f, f)$.

To establish these estimates, first recall [4, p. 157] that for any $m<r, \delta>0$, there is a $C(\delta)$ such that $\left\|D^{m} f\right\|<\delta\left\|D^{r} f\right\|+C(\delta)\|f\|$. Next note that

$$
\begin{aligned}
\operatorname{Re}\left(\left[D^{r} A_{2 r} D^{r}\right] f, f\right) & =\operatorname{Re}(-1)^{r}\left(A_{2 r} D^{r} f, D^{r} f\right) \\
& =\int_{0}^{1}\left(D^{r} f\right)^{*}\left\{\operatorname{Re} A_{2 r} D^{r} f\right\}>c\left\|D^{r} f\right\|^{2} .
\end{aligned}
$$

Thus by choosing $\mu$ large and $\delta$ small we can force the inequality

$$
\operatorname{Re}([L+\mu I] f, f)>c_{1}\left[\left\|D^{r} f\right\|^{2}+\|f\|^{2}\right]
$$

for any $0<c_{1}<c$. Similarly, $|\operatorname{Im}([L+\mu I] f, f)|=|\operatorname{Im}(L f, f)|<c_{3}\left[\left\|D^{r} f\right\|^{2}+\|f\|^{2}\right]$ for some $c_{3}>0$. If we let $c_{2}=c_{3} / c_{1}$

$$
|\operatorname{Im}([L+\mu I] f, f)|<c_{2} \operatorname{Re}([L+\mu I] f, f)
$$

results, which establishes the claim. 
Let $\mathfrak{L}_{0}$ denote the operator on $\oplus^{k} L^{2}\left[U\left(x_{0}\right)\right]$ with domain consisting of the $n$ times continuously differentiable $k$-vector valued functions $f$ with support in $U\left(x_{0}\right)$, and such that $\mathfrak{L}_{0} f=(L+\mu I) f$ for $f \in D\left(\mathscr{L}_{0}\right)$. By $\mathfrak{L}_{0}^{+}$we mean the operator with $D\left(\mathfrak{L}_{0}\right)$ and

$$
\mathfrak{L}_{0}^{+} f=\sum_{j=0}^{n}(-1)^{j} D^{j} A_{j}^{*}(x) f+\bar{\mu} f=(L+\mu I)^{+} f .
$$

If $\mathfrak{L}_{\max }$ is the maximal regular operator associated with $L+\mu I$, then $\left(\mathfrak{C}_{0}\right)^{*}=\mathfrak{L}_{\max }^{+}$ and $\left(\mathfrak{L}_{0}^{+}\right)^{*}=\mathfrak{L}_{\max }([2]$ or $[4])$.

The inequality (ii) means that $\mathscr{L}_{0}$ is sectorial in the sense of Kato [6, p. 318]. The inequality (i) guarantees that zero is not in the numerical range of $\mathfrak{L}_{0}$. It follows (Kato [6, pp. 318 and 322]) that $\mathfrak{L}_{0}$ has a closed invertible extension $\mathcal{L}$ preserving (ii); hence $\mathfrak{L}^{-1}$ has real part greater than or equal to zero. Further (Kato [6, p. 323, Theorem 2.5 and Corollary 2.4]) $\mathcal{L}^{*}$ is an extension of $\mathfrak{E}_{0}^{+}$; thus $\mathcal{L}$ is a restriction of $\mathcal{L}_{\max }$.

Since $\mathcal{L}$ is an ordinary differential operator of order at least $2, \mathfrak{L}^{-1}$ is trace class ([1], [7]). A theorem of Lidskii ([3, p. 231], [7, p. 149]) shows that the generalized eigenvectors of $\mathfrak{L}^{-1}$ have dense span in $L^{2}\left[U\left(x_{0}\right)\right]$.

Suppose that $\left[\mathfrak{Q}^{-1}-\lambda I\right]^{m} x=0$. Notice that $\lambda \neq 0$. For some constants $c_{j}$ we have $x=\sum_{j=1}^{m} c_{j} \mathfrak{L}^{-j} x$, which implies that $x$ is in the domain of every power of $\mathfrak{L}$. Thus $\mathfrak{L}^{m}\left[\mathfrak{L}^{-1}-\lambda I\right]^{m} x=0=(I-\lambda \mathscr{L})^{m} x=(\mathfrak{L}-I / \lambda)^{m} x$. Consequently, the span of the generalized eigenfunctions of $\mathcal{L}$ are dense in $\bigoplus^{k} L^{2}\left[U\left(x_{0}\right)\right]$. Since $D(\mathscr{L}) \subset$ $D\left(L_{\max }\right)$, it follows that the generalized eigenfunctions of $L_{\max }$ have dense span. By Lemma 2 we are done.

The last result is a corollary to the proof of Theorem 1. If $m$ is a positive integer and $J$ is a compact interval, then $C^{m}([J])$ is a Banach space when equipped with the norm $\|f\|=\sum_{k=0}^{m} \sup _{x \in J}\left|f^{(k)}(x)\right|$.

CoROLlaRY 6. For every $m$ and every $x_{0} \in(0,1)$ there is a compact interval $J\left(x_{0}\right)$, containing a neighborhood of $x_{0}$ such that $S$ restricted to $J\left(x_{0}\right)$ is dense in $C^{m}\left[J\left(x_{0}\right)\right]$.

Proof. As we noted in the proof of Theorem 1, we can assume without loss of generality that $L$ has order $r, m<r$, and that for some positive constants $c, k$ we have $\|L f\|>C\left[\left\|D^{r} f\right\|+\|f\|\right]$ for all $f \in C^{\infty}$ which are supported in some fixed neighborhood $U\left(x_{0}\right)$ of $x_{0}$. If $J\left(x_{0}\right)$ is a compact subinterval of $U\left(x_{0}\right)$ containing $x_{0}$ in its interior, then we also have $C\left[\left\|D^{r} f\right\|+\|f\|\right]>k\|f\|_{C{ }^{m}\left[J\left(x_{0}\right)\right]}$.

Let $\varepsilon>0$ and choose a linear combination $\Sigma a_{i} \chi_{i}$ of eigenfunctions $\chi_{i}$ of $L$ such that $L \chi_{i}=\lambda_{i} \chi_{i}, \lambda_{i} \neq 0$, and $\left\|\Sigma a_{i} \chi_{i}-L f\right\|_{L^{2}\left\{U\left(x_{0}\right)\right\}}<k \varepsilon$. Then

$$
\left\|L\left\{\sum a_{i} x_{i} / \lambda_{i}-f\right\}\right\|_{L^{2}\left[U\left(x_{0}\right)\right]}<k \varepsilon,
$$

and thus

$$
\left\|\sum a_{i} \frac{\chi_{i}}{\lambda_{i}}-f\right\|_{C^{m}\left[J\left(x_{0}\right)\right]}<\varepsilon .
$$

Since $f$ was any $C^{\infty}$ function supported in $U\left(x_{0}\right)$, and the restrictions of these functions to $J\left(x_{0}\right)$ are dense in $C^{m}\left[J\left(x_{0}\right)\right]$, we are finished. 


\section{REFERENCES}

1. R. Carlson, Expansions associated with non-self-adjoint boundary-vahue problems, Proc. Amer. Math. Soc. 73 (1979), 173-179.

2. E. A. Coddington and A. Dijksma, Adjoint subspaces in Banach spaces, with applications to ordinary differential subspaces, Ann. Mat. Pura Appl. (to appear).

3. I. C. Gohberg and M. G. Krein, Introduction to the theory of linear nonselfadjoint operators, Transl. Math. Monographs, vol. 18, Amer. Math. Soc., Providence, R. I., 1969.

4. S. Goldberg Unbounded linear operators, MaGraw-Hill, New York, 1966.

5. G. H. Hardy and E. M. Wright, An introduction to the theory of mambers, Oxford Univ. Press, Oxford, 1956.

6. T. Kato, Perturbation theory for linear operators, Springer-Verlag, New York, 1966.

7. J. Ringrose, Compact non-self-adjoint operators, Van Nostrand Reinhold, London, 1971.

Departmient of Mathibatics, Universtty of Utah, Salt Lake CtTy, Utah 84112 